\title{
Magnetization reversal of ferromagnetic nanowires studied by magnetic force microscopy
}

\author{
T. G. Sorop, ${ }^{1}$ C. Untiedt, ${ }^{1}$ F. Luis, ${ }^{2}$ M. Kröll, ${ }^{3}$ M. Raşa,${ }^{4}$ and L. J. de Jongh ${ }^{1, *}$ \\ ${ }^{1}$ Kamerlingh Onnes Laboratory, Leiden University, 2300 RA Leiden, The Netherlands \\ ${ }^{2}$ Instituto de Ciencia de Materiales de Aragon, Universidad de Zaragoza-C.S.I.C., 50009 Zaragoza, Spain \\ ${ }^{3}$ Physics Department, Trinity College Dublin, Dublin, Ireland \\ ${ }^{4}$ Van't Hoff Laboratory for Physical and Colloid Chemistry, Debye Institute, Utrecht University, Padualaan 8 , \\ 3584 CH Utrecht, The Netherlands
}

(Received 6 June 2002; revised manuscript received 23 September 2002; published 9 January 2003)

\begin{abstract}
The magnetization reversal of two-dimensional arrays of parallel ferromagnetic Fe nanowires embedded in nanoporous alumina templates has been studied. By combining bulk magnetization measurements (superconducting quantum interference device magnetometry) with field-dependent magnetic force microscopy (MFM), we have been able to decompose the macroscopic hysteresis loop in terms of the irreversible magnetic responses of individual nanowires. The latter are found to behave as monodomain ferromagnetic needles, with hysteresis loops displaced (asymmetric) as a consequence of the strong dipolar interactions between them. The application of field-dependent MFM provides a microscopic method to obtain the hysteresis curve of the array, by simply registering the fraction of up and down magnetized wires as a function of applied field. The observed deviations from the rectangular shape of the macroscopic hysteresis loop of the array can be ascribed to the spatial variation of the dipolar field through the inhomogeneously filled membrane. The system studied proves to be an excellent example of the two-dimensional classical Preisach model, well known from the field of hysteresis modeling and micromagnetism.
\end{abstract}

DOI: 10.1103/PhysRevB.67.014402

PACS number(s): 75.75.+a, 75.60.Jk, 68.37.Rt

\section{INTRODUCTION}

Magnetic nanoparticles or nanowires are attracting much attention because they offer the opportunity to study magnetism in between the atomic and bulk limits ${ }^{1}$ and because ordered arrays of ferromagnetic particles or wires are of potential interest for applications such as ultra-high-density magnetic recording devices. ${ }^{2}$ In the last decade, the introduction of new experimental techniques, such as electron holography, ${ }^{3}$ micro-SQUID magnetometry, ${ }^{4}$ and magnetic force microscopy, ${ }^{5}$ has provided direct ways to probe the magnetic properties of individual nanoparticles. These studies show that the magnetization reversal of small, singledomain particles is rather well described by the classical Néel-Brown model for rotation in unison. ${ }^{6}$ The situation is, however, different for elongated particles ${ }^{5,7,8}$ or magnetic wires, ${ }^{4,9,10}$ for which flipping of the magnetization appears to proceed via an inhomogeneous reversal mode, such as curling or nucleation of a reversed domain followed by the propagation of the domain wall. In addition, experiments ${ }^{4,8-10}$ as well as theoretical models ${ }^{11}$ appear to agree on the fact that nucleation occurs preferentially at the wire's ends or at defects. Furthermore, when the particles become more and more densely packed in ordered arrays, the magnetization reversal processes become affected by interparticle interactions. Depending on the symmetry of the array, these may either enhance or reduce the switching fields of the individual particles. On the basis of the above, it is clearly of prime importance to study the switching properties of individual wires as well as their mutual interactions.

A relatively simple procedure to fabricate arrays of metallic nanowires is to start from nanoporous materials as templates and fill the pores with metal by electrochemical methods. ${ }^{12}$ Track-etched polymer membranes, anodized alu- minum films, and nanoporous silica phases are attractive examples in this respect. A particular advantage of the alumina templates is that the nanopores all run in parallel, with the long axis perpendicular to the film surface, and with aspect ratio's that can be as high as $10^{3}$. Although these nanoporous films have been known for quite some time, ${ }^{13}$ recent research in self-organized nanostructured materials has revived interest in them and several groups are trying to optimize their structural properties as regards homogeneity of pore size, pore distance, pore filling, and regularity of the superlattice. ${ }^{14,15}$ Pore diameters now extend from well below 10 $\mathrm{nm}$ up to several hundreds of nanometers, with lengths of the order of $1-100 \mu \mathrm{m}$.

In our group we have previously prepared $\mathrm{Fe}, \mathrm{Co}$, and $\mathrm{Ni}$ alumites and studied their magnetic properties. ${ }^{10}$ Pore diameters $D_{p}$ varied from $50 \mathrm{~nm}$ down to $6 \mathrm{~nm}$, i.e., of the same order and even lower than the values of the domain-wall width $\lambda_{w}=2\left(A_{J} / K_{s}\right)^{1 / 2}$, where $A_{J}$ and $K_{s}$ are, respectively, the exchange constant and the shape anisotropy. For the Fe and $\mathrm{Ni}$ nanowires, one has $\lambda_{w} \simeq 6-8 \mathrm{~nm}$ and $22-28 \mathrm{~nm}$, respectively. ${ }^{16}$ From the size dependence of the lowtemperature coercive field $B_{c}$, as measured at $5 \mathrm{~K}$, a clear deviation from the curling mode prediction was found for $D_{p}<\sqrt{\pi} \lambda_{w}$, with $B_{c}$ levelling off to a value of about onethird of the prediction for rotation in unison (the so-called Stoner-Wohlfarth model) at lowest $D_{p}$. This appears to be in good agreement with the theoretical prediction of Braun ${ }^{11}$ for nonuniform magnetization reversal by means of solitonic excitations near the ends of the wires. The small values observed for the involved activation energy, and its proportionality to the cross section instead of the volume of the wires, confirm such an interpretation. ${ }^{10}$

The aim of the present work is to further investigate the manner in which the dipole-dipole interactions modify the 
Stoner-Wohlfarth magnetic response of a single wire, and whether these interactions determine the distribution of switching fields and, therefore, the overall shape of the macroscopic hysteresis loop. To achieve this, we combine standard bulk magnetization measurements [using superconducting quantum interference device (SQUID) magnetometry] with microscopic techniques such as atomic and magnetic force microscopy (AFM/MFM). Recent reports by other groups ${ }^{15,17-20}$ have shown such an approach to be very fruitful indeed. When compared to these studies, the nanowires prepared in alumite membranes are ideal systems for investigating the sole effect of interactions, i.e., separated from other effects such as thermal relaxation or inhomogeneous remanent states. Because of the large aspect ratios $(>60)$ of our wires, they have a remanence close to unity and reverse their magnetization via irreversible jumps. These properties ensure, as our results indeed show, that the average magnetization of an array can be obtained by counting the number of wires with magnetization up and down in MFM pictures. Furthermore, thermal effects (reduction of the coercive field and remanence) are greatly suppressed by the large volume (and the related activation energies) of the particles. ${ }^{10} \mathrm{We}$ show that our arrays of magnetic wires behave exactly as predicted by Preisach-type models, in which the StonerWohlfarth response (square hysteresis loop) is modified by interactions. This simplifies considerably the interpretation and enables us to draw conclusions with a minimum modeling of the data. Last but not least, the field created by the tip has an appreciable magnitude only over the small region of the wires (the apex) that is closest to it. Therefore, the experimental situation is ideal to use MFM as a noninvasive probe to measure the hysteresis loop, as our experiments show. The comparison between hysteresis loops of single wires in different regions of the sample and the macroscopic loop indicates that the dipolar interactions between wires are not only responsible for the shearing of the hysteresis loop but also provide the main contribution to the observed distribution of switching fields. This distribution originates from the different possible environments of the wires in the inhomogeneously filled array, and from the different sizes of the filled regions in a sample.

The outline of this paper is as follows. In the following section we describe experimental details. Section III is devoted to the presentation and discussion of the experiments. Our main conclusions are summarized in Sec. IV.

\section{EXPERIMENTAL DETAILS}

Fe-filled alumite membranes were prepared and characterized as described in Ref. 10. The morphology and size of the pores were determined by scanning (SEM) and transmission electron microscopy (TEM), and by AFM. Top-view AFM and top-view and side-view SEM pictures (see Fig. 1) confirm that the pores are uniform in size, are well separated, and form a regular hexagonal structure. For this work, we chose samples with $D_{p}=50 \mathrm{~nm}$ (interpore distance $D_{i n t}$ $=85 \mathrm{~nm}$ ), since for such a value the hexagonal order of the two-dimensional (2D) superlattice is known to extend over large distances. Moreover, for smaller sizes the thermal re-
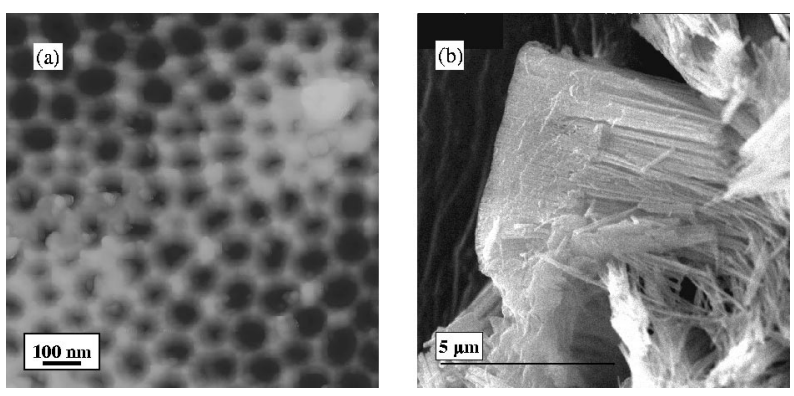

FIG. 1. (a) Typical AFM picture taken on an area of about $900 \times 900 \mathrm{~nm}$ at the top of the empty alumina membrane, (b) SEM picture with a size of $10 \times 10 \mu \mathrm{m}^{2} \mathrm{~nm}$ after the alumina membrane has been partially dissolved. One may distinguish a large bundle of wires originating from a region of filled pores with highly uniform, straight, and parallel Fe nanowires.

laxation effects become important. ${ }^{10}$ Just before the AFM and MFM measurements were performed, the remaining $\mathrm{Al}$ layer as well as the barrier layer located underneath the bottom of the wires were eliminated from the film by chemical etching. The pictures presented below correspond to the thus obtained bottom surface, which turns out to be flatter than the upper surface for all samples. Although we only present data obtained for two representative samples (labeled Fe-1 and Fe-2), similar results were obtained for other samples with different filling fractions and/or aspect ratios of the wires. Cross-sectional side-view TEM and SEM pictures [cf. Fig. 1(b)] show that each alumite membrane is divided into regions of pores completely filled from top to bottom, separated by regions of completely empty pores. The length of the wires is quite uniform, typically of the order of few micrometers (3-6 $\mu \mathrm{m}$ for the investigated samples). Mössbauer spectroscopy measurements ${ }^{10}$ confirm that the Fe is predominantly in the $\alpha$-Fe phase, with a magnetic moment per atom equal to the bulk value. They also show that the needles are apparently monodomain with the easy magnetization direction along the wire axis as a consequence of the shape anisotropy. Only a few percent of the Fe is found to be oxidized, likely corresponding with the surface layers at the cylinder walls. Recent extended x-ray-absorption finestructure and $x$-ray diffraction studies on our samples ${ }^{21}$ are consistent with these conclusions.

The magnetization of about $3 \mathrm{mg}$ of each membrane was measured with a commercial SQUID magnetometer. For the data shown here the magnetic field was always parallel to the wires (for the perpendicular data see Ref. 10). A commercial NanoScope III (Digital Instruments) scanning probe system with a MultiMode ${ }^{\mathrm{TM}}$ microscope was used for the AFM/ MFM measurements. The probes consist of cantilevers with single-crystal silicon tips coated with a thin magnetic film (Co-Pt-Cr alloy). These high coercivity tips, with $B_{c}$ $\geqslant 0.5 \mathrm{~T}$, have a conical shape, with an end that can be modeled as a sphere with radius $40-60 \mathrm{~nm}$. They were premagnetized to saturation. Although it is rather difficult to give an accurate value for the field exerted by the magnetic tip, we estimated it to be a few tens of millitesla for the distances involved. As discussed below, we found no evidence for an invasive magnetic effect of the tips. 


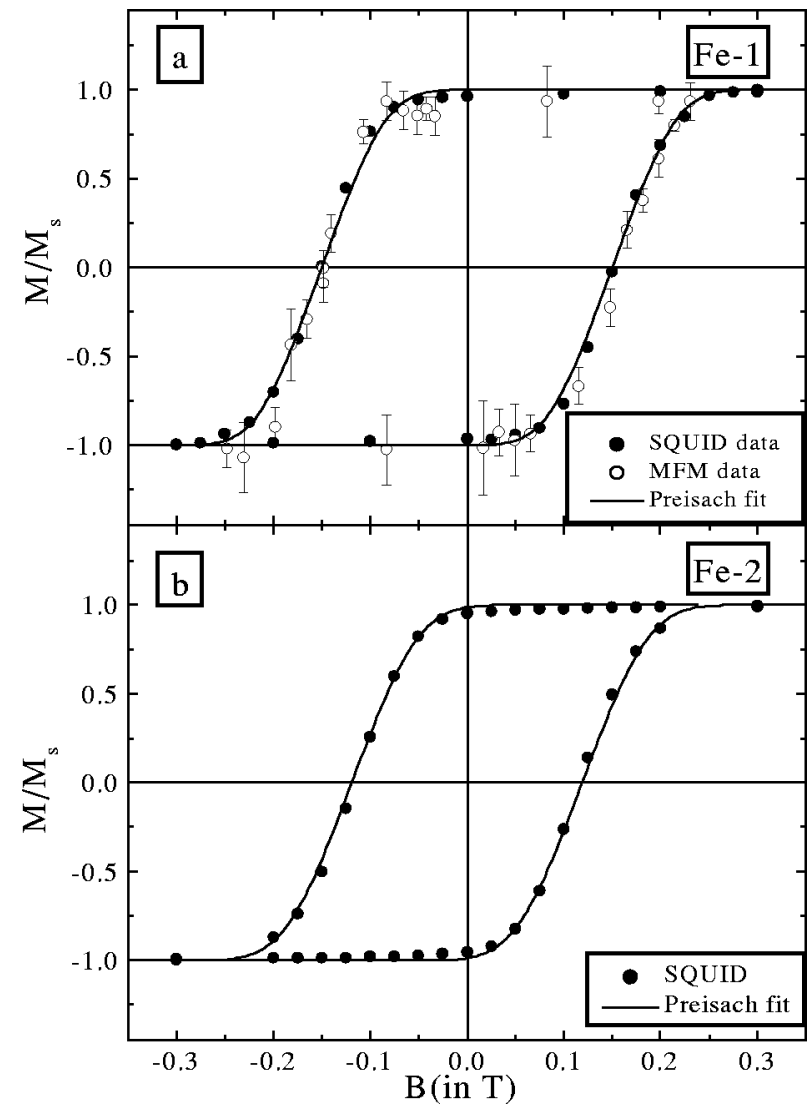

FIG. 2. Hysteresis loops of samples (a) Fe-1 and (b) Fe-2 measured at room temperature with the SQUID magnetometer (filled dots). Values found for the coercive field are $B_{c}=0.151 \mathrm{~T}(\mathrm{Fe}-1)$ and $B_{c}=0.119 \mathrm{~T}(\mathrm{Fe}-2)$, respectively. The included open circles for $\mathrm{Fe}-1$ were obtained from MFM pictures taken on this sample, as described in the text. The full line in both panels is the result of the fit with a Preisach model using parameters $p=0.03$ and $\sigma_{B}$ $=0.03 \mathrm{~T}(\mathrm{Fe}-1)$ and $p=0.03$ and $\sigma_{B}=0.035 \mathrm{~T}(\mathrm{Fe}-2)($ see the text $)$.

The MFM images were recorded using the Digital Instruments Dynamic Lift Mode option, which allows to image relatively weak but long-range magnetic interactions, while minimizing the influence of the topography. In order to perform MFM experiments in an external magnetic field, a home-built coil (similar as in Ref. 22) with a $5 \mathrm{~mm}$ internal diameter and a $15 \mathrm{~mm}$ external diameter was mounted on the scanner. The coil could generate field pulses up to $0.5 \mathrm{~T}$, with a $160 \mu$ s rise time and a relaxation time of about $170 \mu \mathrm{s}$. The field was applied parallel to the long axis of the wires $(z)$ and is constant within less than 5\% over $6 \mathrm{~mm}$ along this direction. Before each scan, a short pulse corresponding to the target field value was first applied, with the magnetic tip retracted. After a few seconds, the magnetic tip was then engaged to the sample and the AFM/MFM pictures were recorded.

\section{RESULTS AND DISCUSSION}

Figure 2 shows the hysteresis loops of sample Fe-1 and sample Fe-2 (filled points) as measured at room temperature by SQUID magnetometry, with the field along the wire axis.
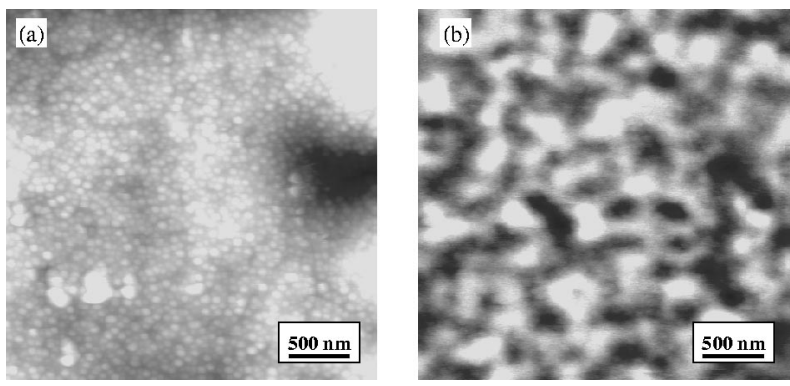

FIG. 3. Typical AFM/MFM picture for sample Fe-2. The left picture is the topography (height image) and the right is the magnetic contrast (phase image).

One may observe a high ratio of remanence to saturation (i.e., squareness), $M_{r} / M_{s} \simeq 0.97$. This indicates that the reversible part of the magnetization is very small, as expected for high aspect-ratio wires. Moreover, $M_{r} / M_{s}$ at room temperature does not deviate much from its value at $5 \mathrm{~K}$, which shows that even at $300 \mathrm{~K}$ all wires remain in the initial state after the saturating magnetic field is removed. Apparently, the activation barriers for the irreversible switching of the magnetic moments are high enough that thermally activated relaxation can be almost completely neglected. Only after a sizable magnetic field is applied in the opposite direction does $M$ start to decrease. Since we wish to compare the macroscopic SQUID measurements with the results obtained with pulsed fields from the MFM pictures, we also measured the hysteresis loop of the remanent magnetization $M_{r}(B)$ with the SQUID by setting the field at a given value and then switching it to zero again. The so-obtained $M_{r}(B)$ is found to be very close to the magnetization loop as measured in the usual way, which confirms that the magnetization changes mainly via irreversible flips. In what follows, we shall show that the macroscopic hysteresis loop can be reproduced by just summing up the responses of individual wires as obtained from MFM measurements. The corresponding data points are included already in Fig. 2.

In Fig. 3, we give an example of the AFM/MFM pictures of sample $\mathrm{Fe}-2$, corresponding to a region of about 3 $\times 3 \mu \mathrm{m}^{2}$. It can be seen that the topography (left) and the magnetic contrast (right) are well separated indeed. Both pictures were recorded at room temperature after a saturating magnetic-field pulse of $0.5 \mathrm{~T}$ had been applied. The results show that the surface investigated (bottom of the sample) is not perfectly flat, probably as a result of nonuniform chemical etching of the barrier layer. The MFM picture, obtained by scanning the same area at a height of $40 \mathrm{~nm}$ above the surface of the sample, is related to the magnetic signal. Since not all the pores are filled with Fe, the wires appear in bright colors and the empty pores in dark (all wires have the same color because the pulse field applied was high enough to saturate the sample magnetization and since $M_{r} / M_{s} \simeq 1$, cf. Fig. 2).

As the external field was varied, we observed no change in the topographic images but only in the magnetic ones, confirming that the magnetic images are not appreciably influenced by the topography. As a consequence, when discussing the response of the wires as a function of field, we shall 

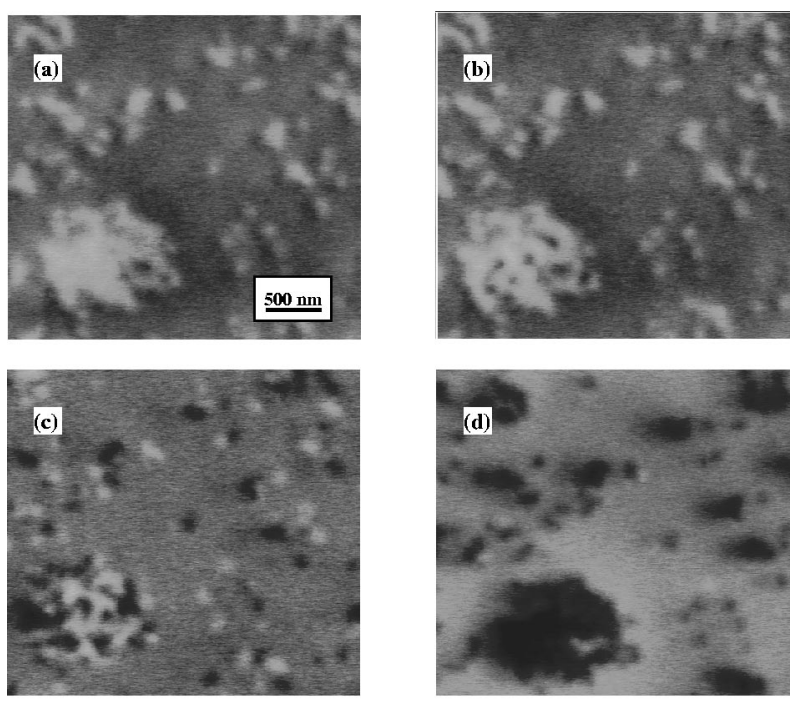

(e)

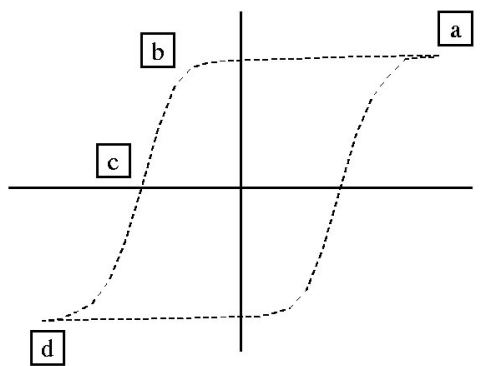

FIG. 4. Succession of MFM pictures in the same area of sample Fe-1. The scan size is $3.5 \times 3.5 \mu \mathrm{m}^{2}$ and the scan height is $35 \mathrm{~nm}$. The applied magnetic field is varied as follows: (a) $B=+0.23 \mathrm{~T}$ and in opposite direction (b) $B=-0.08 \mathrm{~T}$, (c) $B=-0.16 \mathrm{~T}$, (d) $B=-0.23 \mathrm{~T}$; (e) the points (a) $-(\mathrm{d})$ are marked in the hysteresis loop.

restrict to the magnetic images. Figure 4 shows pictures taken for sample Fe-1, which has a smaller filling fraction of the pores, making it easier to observe the switching of the magnetization of individual wires. We point out that, although the magnetic contrast of the empty regions in Fig. 4 also changes gradually and uniformly with the field, this is only due to the change of the average magnetization of the sample, which modifies the average contrast. This overall variation can easily be separated from the rapid and localized changes of contrast observed in the regions containing the magnetic wires.

The series of pictures in Fig. 4 was taken on the same region $\left(3.5 \times 3.5 \mu \mathrm{m}^{2}\right)$ for different amplitudes of the pulsed magnetic field. The experimental procedure was as follows. We first applied a pulsed field of $+0.23 \mathrm{~T}$ antiparallel to the tip magnetization. Since this field exceeds the saturation field, the magnetic moments of all wires are aligned. For this configuration, in which the magnetization directions of tip and wires are antiparallel, all wires should appear in the same (bright) color, as indeed observed. The darker regions here are the empty pores. Next a hysteresis-type cycle was performed with the pulsed field, following the same procedure as for the measurement of the macroscopic remanent magnetization [the field values used for Figs. 4(a)-4(d) have been indicated on the hysteresis curve in Fig. 4(e)].
The MFM pictures do not show any noticeable change until a field opposite to the tip magnetization of about -0.08 $\mathrm{T}$ is applied [Fig. 4(b)], at which some of the bright spots turn into dark indicating the magnetization reversal of some of the wires. By increasing the amplitude of the magneticfield pulse along the same direction, the number of reversed wires grows gradually. In Fig. 4(c) one may observe that near the average coercive field $\left(B_{c}=0.151 \mathrm{~T}\right)$, the amount of reversed and not-yet-reversed wires has become roughly equal; thus the average magnetization on this microscopic scale is approximately zero, just as expected on the basis of the macroscopic hysteresis curve. Finally, by increasing the field still further, all wires become reversed when $B=-0.23 \mathrm{~T}$ [Fig. 4(d)]. This last picture is just the negative of the first [Fig. 4(a)], and the empty regions now appear as the brightest. As shown in Ref. 1, this is consistent because the magnetization directions of the tip and wires are now parallel. Images taken when performing subsequently the same cycle from $B=$ -0.23 T to $B=+0.23$ T show the same changes of contrast of the individual wires but with opposite colors, as it should.

It thus appears that the MFM pictures indeed provide a means to construct the magnetization curve, namely, by simply counting the number of wires with "up" and "down" directions observed for each applied field. The hysteresis loop obtained by this microscopic technique is compared to the SQUID data in Fig. 2(a), where the magnetization data found from MFM were scaled to the SQUID value at saturation. They show a remarkably good agreement as regards shape, symmetry, and the values of coercive and saturation fields. We note that regions such as those shown in Fig. 4 used to count up and down wires contain typically several hundreds of wires, i.e., enough for reliable statistics. We can therefore conclude that the macroscopic hysteresis loop as measured by conventional magnetometry, is actually composed of the contributions from the irreversibly switching individual nanowires, and that the rounding of the hysteresis loop, as observed even at the lowest temperatures $(5 \mathrm{~K})$, is related to a distribution of switching fields among the wires and not to reversible magnetization processes. Although similar MFM studies were recently performed by other groups on different types of samples, most of these involved particles with (very) much lower aspect ratios. ${ }^{17,20,23,24}$ For wires with such high $(>60)$ aspect ratios as ours, the above result is certainly nontrivial since the AFM/MFM is only sensitive to the ends of the wires, whereas the SQUID measures the volume magnetization. Thus, the apparent agreement between the two measurements confirms the conclusion already drawn from the Mössbauer spectra that the $\mathrm{Fe}$ nanowires are indeed monodomain ferromagnetic needles.

In principle, the observed distribution in the individual switching fields $B_{c}$ can be related either to differences in morphology of the wires or to interwire dipolar interactions. Clearly the variation of $B_{c}(T=0)$ with the dimensions of the wires depends on the actual mechanism that drives the magnetization reversal. ${ }^{11,25}$ According to previous experimental evidence, $, 4,10$ the magnetization reverses by curling for $D_{p}$ $\gg \sqrt{\pi} \lambda_{w}(\simeq 11-14 \mathrm{~nm}$ for $\mathrm{Fe})$, whereas when $D_{p}<\sqrt{\pi} \lambda_{w}$ the reversal involves the nucleation of a small reversed domain at the wire ends. In the first case, $B_{c}(T=0) \propto 1 / D_{p}^{2},{ }^{25}$ 
and it is therefore very sensitive to the size of the wires. By contrast, for nucleation in an infinitely long cylinder ${ }^{11} B_{c}$ is independent of $D_{p}$ and equals the limit for rotation at unison, $\pi M_{s b} / 2 \simeq 1.1 \mathrm{~T}$. This is, however, different for finite cylinders because the magnetostatic energy near the end of the wire lowers the effective anisotropy, thus reducing the energy for nucleation ${ }^{11,16}$ as was indeed observed. ${ }^{10}$ In this case, the coercive field depends weakly on $D_{p}$ but it can be sensitive to the presence of defects near the end of the wires. The wires studied here have $D_{p}$ in the region where the transition between these two limiting situations takes place. ${ }^{10}$ Hinzke and Nowak ${ }^{26}$ have recently predicted that the reversal then resembles the nucleation of a domain at one of the ends of the wire in which the atomic spins in a plane perpendicular to the wire's long axis are not parallel, as for curling. We therefore expect that $B_{c}(T=0)$ depends on $D_{p}$, although not so strongly as for curling. Top-view and side-view SEM/ TEM pictures for our samples (see, for example, Fig. 1) show a quite regular structure, with very long and straight wires and only a small distribution in diameters and length. The fact that the wires stay intact after dissolving the alumina matrix shows that they are not made up of granular particles. The morphology is, therefore, unlikely the only source of broadening. Although thermal fluctuations can also broaden the switching field distribution, this effect is apparently not very important since the shape and width of the hysteresis loop does not change much when cooling from room temperature down to $2 \mathrm{~K}$. As argued below, the dipoledipole interactions between the wires can play a major if not dominant role in the width of the distribution of switching fields. In view of the inhomogeneous filling of the pores in the templates (cf. Figs. 3 and 4), a large spatial variation in the dipolar field felt by the wires is indeed to be expected. When dipolar interactions are important, the response of a magnetic wire thus depends on the magnetic state of its neighbors, i.e., on the magnitude and the orientation of the local dipolar field with respect to direction of the applied field. For a single wire, the hysteresis loop can then become very asymmetric. For a sufficiently large array, the sum of the individual contributions may still produce a symmetric macroscopic hysteresis loop, but it will be substantially broadened. As shown next, MFM experiments confirm this and enable us to separately estimate the distributions in the dipolar fields and in the local switching fields $\left[B_{c}(T=0)\right]$ of the wires.

The effects of the interwire interactions can be evidenced by studying the hysteresis loop of a single wire, or of few coupled wires. In Fig. 5, a series of four MFM images is presented, taken from sample Fe-2 under different applied fields. First a pulsed field of $0.52 \mathrm{~T}$ is applied, large enough to align all the magnetic moments. For convenience, we call it the upward direction since in this initial configuration the magnetization of wires and tip are parallel, all wires show a dark color [Fig. 5(a)]; the bright regions correspond to empty pores only. This picture remains unchanged until a relatively small magnetic field of about $0.05 \mathrm{~T}$ is applied in the opposite downward direction [Fig. 5(b)]. Then one wire is seen to change its color from dark to bright, which shows that it has already flipped in a downward switching field of about
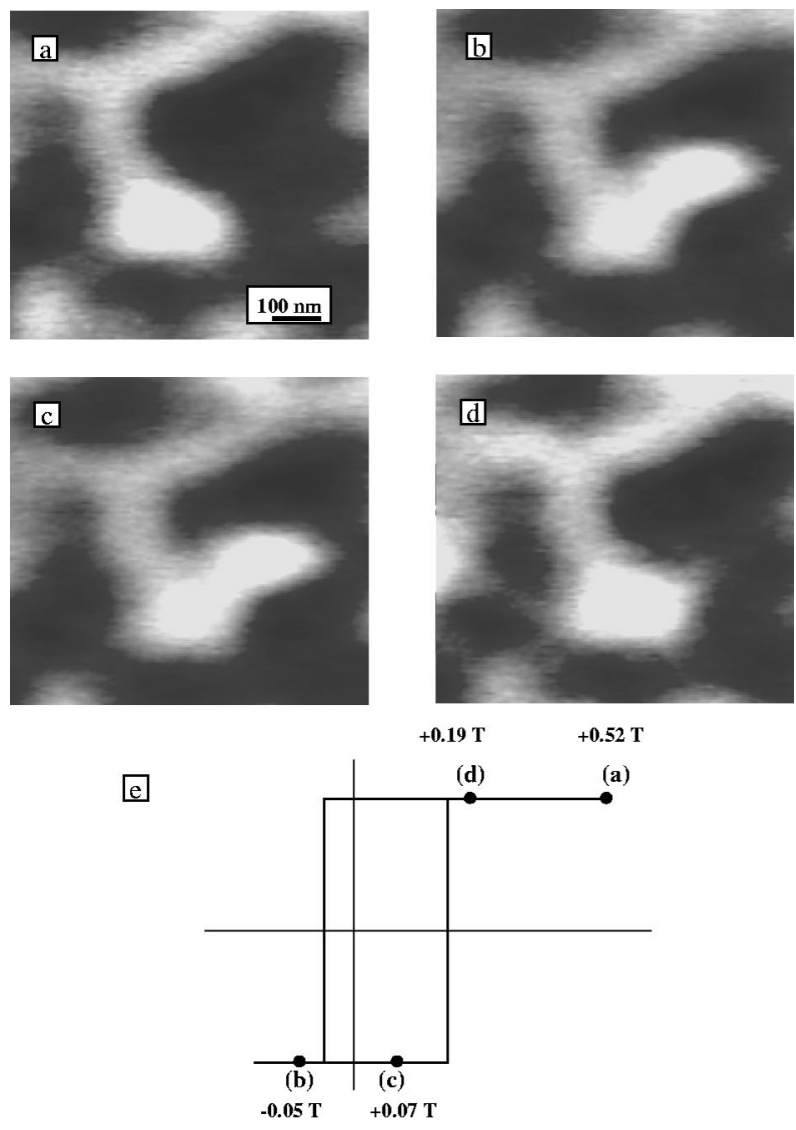

FIG. 5. (a)-(d) Magnetic force microcopy images obtained for sample Fe-2 after applying magnetic-field pulses of different amplitudes. (e) Hysteresis loop of a single wire as obtained from MFM.

$0.05 \mathrm{~T}$, i.e., very much smaller than the average coercive field of the array. We then reversed the direction of the applied field back upward, during which process all wires retain their color. Remarkably, even for an upward field pulse of about $0.07 \mathrm{~T}$, i.e., larger than the switching field observed for downward fields, the reversed wire has not yet switched back [Fig. 5(c)]. In fact, it only does so when a much larger field (at least $0.19 \mathrm{~T}$ ) is applied in the upward direction [Fig. 5(d)]. From comparable results found for 24 different wires in the array, we obtained similar loops, showing that although the switching of the individual wires occurs in a rather narrow field range, the value of the switching field and thus the degree of asymmetry strongly depend on the magnetic history and on the configuration of the surrounding wires. It is of interest to point out that the maximum observed asymmetry of about $0.1 \mathrm{~T}$ is of the same order as the broadening of the macroscopic loop. As anticipated, this asymmetry finds its natural explanation in the dipolar field generated by neighboring wires in the sample. Since this type of interaction is energetically favoring the antiparallel orientation of neighboring wires, the switching field of an individual wire will be different depending on its direction: it will take a higher field to change the direction of a switched wire back from antiparallel to parallel relative to its neighbors.

From the variation in the observed asymmetry of the individual loops, we can conclude that the interwire dipolar 
interactions can vary from $0.01 \mathrm{~T}$ to values as high as $0.1 \mathrm{~T}$, when expressed in terms of an effective field. We may compare this with a simple estimate of the dipolar coupling, following a model used by Ishii and Sato ${ }^{27}$ and by Lodder and co-workers. ${ }^{28}$ To find the magnetostatic energy between two neighboring wires they are replaced by two (physical) dipoles of length $L$ and having monopole charges $\pm\left(\pi D_{p}^{2} / 4\right) M= \pm \mu / L$. Here $M$ is the average magnetization of the wire and $\mu$ is its total magnetic moment. If $D_{\text {int }}$ is the distance between monopoles, the magnetostatic energy for the parallel orientation, $E_{\|}$, is given by $E_{\|}=\left(\mu^{2} / 2 \pi L\right)$ $\times\left[D_{\text {int }}^{-1}-\left(D_{\text {int }}^{2}+L^{2}\right)^{-1 / 2}\right]$, whereas that for the antiparallel orientation is given by $-E_{\|}$. Next, we may equate the difference between parallel and antiparallel orientations, $\Delta E$ $=2 E_{\|}$, to the difference in Zeeman energy, $\mu \Delta B_{s}$, associated with the difference between the switching fields $B_{s}$ needed to reverse the moment of a wire towards the parallel or the antiparallel configurations. If the reference wire is part of a filled region, we may estimate the maximum energy difference involved by switching it with respect to a parallel background of successive shells of neighbors in the hexagonal array. Inserting typical experimental values for $L$ $=3 \mu \mathrm{m}$ (sample Fe-2) and $D_{\text {int }}=85 \mathrm{~nm}$, we obtain for two neighboring wires $\Delta B_{s}=1.3 \mathrm{mT}$. Including contributions from the first 20 neighboring shells, it yields a value for $\Delta B_{s}$ of about $0.13 \mathrm{~T}$. Thus, in spite of the crudeness of the model, we may state that the strong interwire interaction observed is as expected on the basis of just dipolar interactions. We point out that including neighboring shells up to the 20th neighbor already yields a surface area within the array of about $1 \mu \mathrm{m}^{2}$, which approaches the estimated size of the filled regions [cf. Fig. 1(b)]. Dipolar couplings between filled regions are calculated to be quite small $(\simeq 1 \mathrm{mT})$, so they can safely be neglected. It is also clear from the model that wires at the periphery of a filled region will experience a quite different dipolar field as that in the center.

Summing up the magnetic properties of the system of $\mathrm{Fe}$ nanowires embedded in the alumina template we can state that the arrays can be described as consisting of singledomain particles (i.e., the nanowires); the particles have only two possible states for magnetization (up and down); each isolated particle has a square hysteresis loop; the interparticle interaction is magnetostatic, leading to a shift of the individual loops by an interaction field; there is no reversible or apparent reversible component of the magnetization. In fact, all these properties are the assumptions underlying the wellknown classical Preisach model for hysteresis. ${ }^{29}$ According to this model, the statistics of the system determines the shape of the macroscopic major and minor loops.

The majority of real materials do not satisfy the assumptions made in the classical Preisach approach, and modified Preisach models have therefore been proposed (see, for example, Refs. 30 and 31). Recently however, a 2D array of strongly uniaxial magnetic garnet particles ${ }^{32}$ has been shown to be described by the classical Preisach model. In that system the particles are large, i.e., of the order of 40 $\times 40 \mu \mathrm{m}^{2}$, and are separated by $12-\mu \mathrm{m}$ nonmagnetic regions. More recently, the 2D Preisach model was invoked to describe the response of the arrays of low aspect-ratio $\mathrm{Ni}$
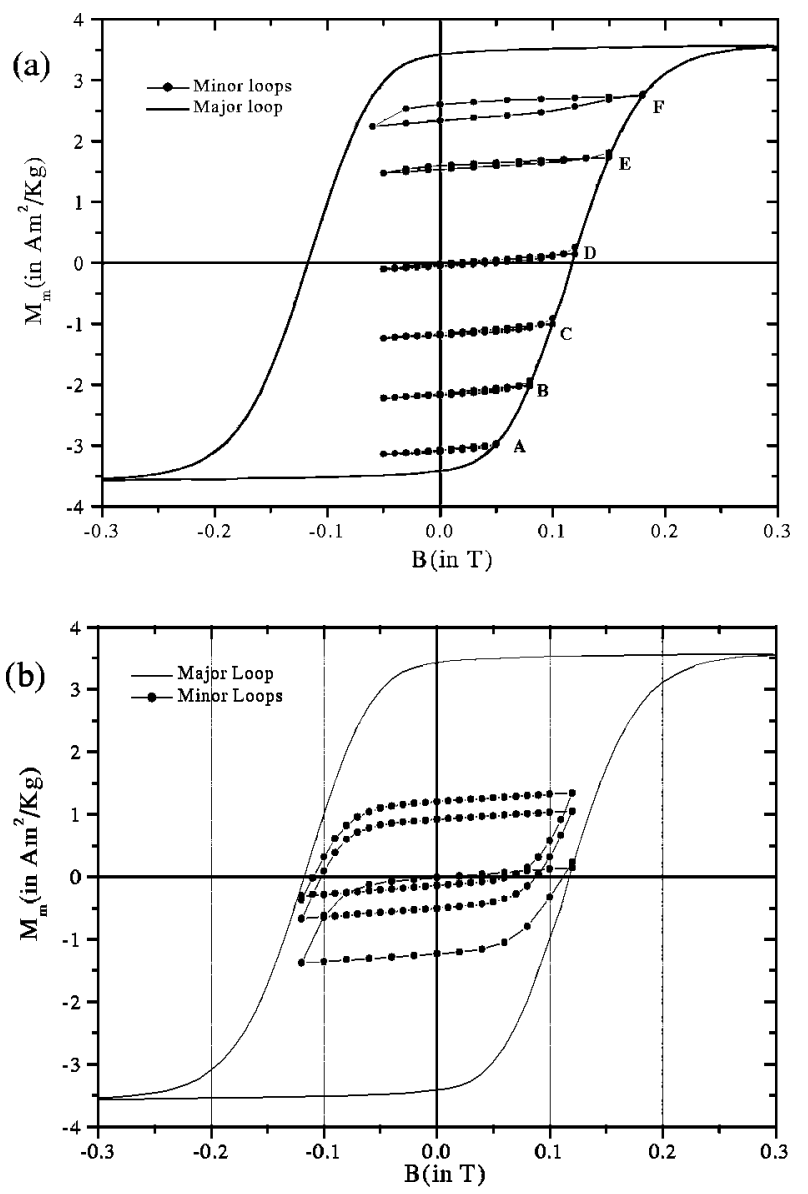

FIG. 6. SQUID magnetization data measured at room temperature, on the sample Fe-2, illustrating (a) the wiping-out property, (b) the congruency property.

particles studied by Ross and co-workers. ${ }^{20}$ We shall now demonstrate in more detail its applicability to our present array, in which the individual particles are smaller, have a much bigger aspect ratio, and also much stronger interactions.

According to the theorem proven mathematically by Mayergoyz, ${ }^{33}$ the "congruency" and the "wiping-out" properties of the minor loops are necessary and sufficient conditions for a hysteretic system to be described by a classical Preisach model. In order to check this, appropriate sets of magnetization data were measured for sample Fe-2 with the SQUID magnetometer. Figure 6 demonstrates that the system has indeed the wiping-out property, i.e., all the minor loops originating from the major loop close at the same point and, in addition, the congruency property, i.e., all the minor loops with identical upper and lower field limits are congruent in the geometrical sense. In Fig. 6(a) a few minor loops are shown that were measured starting from the major loop. If one considers the minor loops initiating in the points $A$ to $F$, it is seen that they all close at the initial value of the magnetization. This means that the variations of the field have erased (wiped-out) the past history. In Fig. 5(b) a few measured minor loops are shown that have the same limiting field $\pm 0.1 \mathrm{~T}$. One can easily see that all the minor loops have the same shape and the same enclosed area. In other words 
they are geometrically congruent. We conclude that, even for our highly diluted (low filling fraction) 2D arrays, the requirements for the Preisach model are met. Apparently, the intrinsic distribution in the interaction arising from incomplete filling does not render the model invalid.

Once that we have shown its applicability, we can use the Preisach model to fit the hysteresis loop for the present arrays and to extract in this way quantitative information on the distribution of switching fields. Since the number (24) of individual wire reversals detected is too limited for reliable statistics, we used the same method as in Ref. 10, but restricted for $T=0$. In order to calculate the dipolar field for these disordered arrays of wires we content ourselves with the simplest approximation and take $B_{d i p}=\mu_{0} p M$, as for a slab where $M$ is the magnetization of the wires in a region and $p$ accounts for the filling fraction of the region and for its finite size. For the sample Fe-1 the model accounts well for the shearing of the hysteresis loop for $p=0.03$, which corresponds to an average dipolar field of about $0.065 \mathrm{~T}$ for a magnetically saturated region, as can be seen from the solid curve in Fig. 2(a). In order to account for the rounding of the hysteresis loop, we have averaged the calculated results by assuming a distribution in the values of the switching field $B_{c}$. For this sample we find a very good agreement for a Gaussian distribution in $B_{c}$ with width $\sigma_{B}=0.030 \mathrm{~T}$. Performing the same analysis with the data of sample Fe-2 [see Fig. 2(b)], we get the value $\sigma_{B}=0.035 \mathrm{~T}$ (in this case the best fit is also obtained for $p=0.03$ ).

We may compare these results to the data on the individual loops coming from the MFM measurements. On basis of the 24 individual loops detected, we obtained the estimates of the average dipolar field and switching field as $B_{\text {dip }} \simeq 0.060 \mathrm{~T}$ and $B_{c} \simeq 0.115 \mathrm{~T}$, whereas the corresponding widths of the distributions were found to be of the order of $0.03 \mathrm{~T}$ for both. These values agree well with those obtained from the fit to the SQUID curves. It is interesting to note that the MFM data enable a separate determination of the distributions in $B_{d i p}$ and $B_{c}$.

\section{CONCLUSIONS}

In this work we have studied the magnetization reversal of $2 \mathrm{D}$ arrays of parallel ferromagnetic Fe nanowires embedded in nanoporous alumina templates. By combining bulk magnetization measurements with field-dependent magnetic force microscopy studies, the macroscopic magnetic response of the array could be decomposed in terms of contributions from individual wires (or sets of few wires). Thereby the important role of dipolar interactions between the wires was put in evidence. In agreement with theory, the hysteresis loops of individual wires are found to be rectangular, with well-defined switching fields. However, since each wire feels a local dipolar field from its neighbors in addition to the applied field, the individual loops are displaced with respect to the origin. As a consequence of the inhomogeneous $\mathrm{Fe}$ filling of the membranes, the dipolar field is spatially varying, so that the loop displacement is also varying from one wire to another. Although the asymmetry of the individual loops is averaged out for the response for the membrane as a whole, the macroscopic hysteresis loop (which should be rectangular in the absence of the interactions) is substantially broadened by the spatial distribution in dipolar fields. This confirms the conclusion stated already in our earlier work ${ }^{10}$ that the observed broadening is not primarily caused by the thermal fluctuations or inhomogeneities in the size and the shapes of the wires.

As part of the analysis, it was confirmed that also for our arrays, field-dependent MFM measurements can provide indeed a microscopic method to measure the magnetization, namely, by simply counting the number of wires with magnetization up and down in the MFM pictures. The hysteresis loop resulting from this surface technique was shown to agree quite well with that obtained by bulk (volume) magnetization measurements. This shows implicitly that the high aspect-ratio $\mathrm{Fe}$ nanowires in these templates are indeed behaving as monodomain ferromagnetic needles that switch their magnetic moments irreversibly from up to down orientations along the wire axis.

Finally, it was shown that, in agreement with the abovementioned properties, the investigated templates can be regarded as an archetypical example of the 2D classical Preisach model, well known in the field of hysteresis modeling.

\section{ACKNOWLEDGMENTS}

This work was part of the research program of the Stichting voor Fundamenteel Onderzoek der Materie (FOM), which is supported by the Nederlandse Organisatie voor Wetenschappelijk Onderzoek (NWO). C.U. and F.L. acknowledge grants funded by the European Union in the framework of its TMR Marie Curie Program. M.K. acknowledges a grant funded by Deutsche Forschungsgemeinschaft (DFG). We gratefully acknowledge the Colloid Physics Stimulation Program, awarded by the Utrecht University to the Debye Research Institute, for the use of the MFM apparatus. We thank Professor A. P. Philipse and A. Morello for their interest and help on the experiments and Professor Domingo González for his many stimulating comments.
*Email address: dejongh@phys.leidenuniv.nl

${ }^{1}$ Jing Shi, S. Gider, K. Babcock, and D.D. Awschalom, Science 271, 937 (1996).

${ }^{2}$ J.L. Simonds, Phys. Today 48 (4), 26 (1995).

${ }^{3}$ C. Beeli, B. Doudin, and P. Stadelmann, Phys. Rev. Lett. 75, 4630 (1995).

${ }^{4}$ W. Wernsdorfer, B. Doudin, D. Mailly, K. Hasselbach, A. Benoit, J. Meier, J.-Ph. Ansermet, and B. Barbara, Phys. Rev. Lett. 77,
1873 (1996).

${ }^{5}$ M. Lederman, S. Schultz, and M. Ozaki, Phys. Rev. Lett. 73, 1986 (1994).

${ }^{6}$ W. Wernsdorfer et al., Phys. Rev. Lett. 78, 1791 (1997); Phys. Rev. B 55, 11552 (1997).

${ }^{7}$ A.D. Kent, S. von Molnar, S. Gider, and D.D. Awschalom, J. Appl. Phys. 76, 6656 (1994).

${ }^{8}$ S. Wirth, M. Field, D.D. Awschalom, and S. von Molnar, Phys. 
Rev. B 57, R14 028 (1998); S. Wirth, S. von Molnar, M. Field, and D.D. Awschalom, J. Appl. Phys. 85, 5249 (1999); S. Wirth, A. Anane, and S. von Molnar, Phys. Rev. B 63, 012402 (2000).

${ }^{9}$ R. Ferré, K. Ounadjela, J.M. George, L. Piraux, and S. Dubois, Phys. Rev. B 56, 14066 (1997).

${ }^{10}$ P.M. Paulus, F. Luis, M. Kröll, G. Schmid, and L.J. de Jongh, J. Magn. Magn. Mater. 224, 180 (2001).

${ }^{11}$ H.-B. Braun, Phys. Rev. Lett. 71, 3557 (1993); J. Appl. Phys. 76, 6310 (1994); Phys. Rev. B 50, 16501 (1994); J. Appl. Phys. 85, 6172 (1999).

${ }^{12}$ A. Fert and L. Piraux, J. Magn. Magn. Mater. 200, 338 (1999).

${ }^{13}$ J.W. Diggle, T.C. Downie, and G.W. Goulding, Chem. Rev. 69, 365 (1969); J.P. O’Sullivan and G.C. Wood, Proc. R. Soc. London, Ser. A 317, 511 (1970).

${ }^{14}$ H. Masuda and K. Fukuda, Science 268, 1466 (1995); A.J. Yin, J. Li, W. Jian, A.J. Bennet, and J.M. Xu, Appl. Phys. Lett. 79, 1039 (2001); K. Nielsch, F. Müller, A.P. Li, and U. Gösele, Adv. Mater. 12, 582 (2000).

${ }^{15}$ K. Nielsch, R.B. Wehrspohn, J. Barthel, J. Kirschner, U. Gösele, S.F. Fischer, and H. Kronmüller, Appl. Phys. Lett. 79, 1360 (2001).

${ }^{16}$ M.E. Schabes, J. Magn. Magn. Mater. 95, 249 (1991).

${ }^{17}$ C.A. Ross et al., J. Appl. Phys. 89, 1310 (2001).

${ }^{18}$ L. Belliard, J. Miltat, A. Thiaville, S. Dubois, J.L. Duvail, and L. Piraux, J. Magn. Magn. Mater. 190, 1 (1998).

${ }^{19}$ R. O’Barr and S. Schultz, J. Appl. Phys. 81, 5458 (1997).
${ }^{20}$ M. Hwang, M.C. Abraham, T.A. Savas, Henry I. Smith, R.J. Ram, and C.A. Ross, J. Appl. Phys. 87, 5108 (2000); C.A. Ross et al., Phys. Rev. B 65, 144417 (2002).

${ }^{21}$ R.E. Benfield, D. Grandjean, J.C. Dore, Z. Wu, M. Kröll, T. Sawitowski, and G. Schmid, Eur. Phys. J. D 16, 399 (2001).

${ }^{22}$ S. Manalis, K. Babcock, J. Massie, V. Elings, and M. Dugas, Appl. Phys. Lett. 66, 2585 (1995).

${ }^{23}$ G.A. Gibson and S. Schultz, J. Appl. Phys. 73, 4516 (1993); M. Lederman, G.A. Gibson, and S. Schultz, ibid. 73, 6961 (1993).

${ }^{24}$ K. Nielsch, R. B. Wehrspohn, J. Barthel, J. Kirschner, S. F. Fischer, H. Kronmüller, T. Schweinböck, D. Weiss, and U. Gösele, J. Magn. Magn. Mater. 249, 234 (2002).

${ }^{25}$ E.H. Frei, S. Shtrikman, and D. Treves, Phys. Rev. 106, 446 (1957).

${ }^{26}$ D. Hinzke and U. Nowak, J. Magn. Magn. Mater. 221, 365 (2000).

${ }^{27}$ Y. Ishii and M. Sato, J. Magn. Magn. Mater. 82, 309 (1989).

${ }^{28}$ E.O. Samwell, P.R. Bissell, and J.C. Lodder, J. Magn. Magn. Mater. 115, 327 (1992).

${ }^{29}$ F. Preisach, Z. Phys. 94, 277 (1935).

${ }^{30}$ I.D. Mayergoyz and G. Friedman, J. Appl. Phys. 61, 4022 (1987).

${ }^{31}$ F. Vajda and E. Della Torre, IEEE Trans. Magn. 27, 4757 (1991).

${ }^{32}$ M. Pardavi-Horvath, G. Zheng, and G. Vertesy, Physica B 233, 287 (1997).

${ }^{33}$ I.D. Mayergoyz, Phys. Rev. Lett. 56, 1518 (1986). 